\title{
Random Spectral Phase Noise Effect on the Temporal Contrast of Ultra-High Intensity Laser Pulse
}

\author{
Hiromitsu KIRIYAMA, ${ }^{1}$ Yuji MASHIBA,,${ }^{1,2}$ Yasuhiro MIYASAKA, ${ }^{1}$ and Makoto R. ASAKAWA ${ }^{2}$ \\ ${ }^{1}$ Kansai Photon Science Institute, National Institute for Quantum and Radiological Science and Technology, \\ 8-1-7 Umamidai, Kizugawa, Kyoto 619-0215 \\ ${ }^{2}$ Faculity of Science and Engineering, Kansai University, 3-3-35 Yamate-cho, Suita, Osaka 564-8680
}

(Received December 1, 2017)

\begin{abstract}
We report on a detailed analysis of the influence of the random spectral phase noise on temporal contrast of an ultra-high intensity laser pulse. We accurately evaluate the impact of stretcher and compressor optics on the contrast pedestal of the ultra-high intensity laser pulse by precise quantitative characterization of the optics surface. The surface roughness of tens of nanometers on the stretcher and compressor optics causes random spectral phase noise, which reduce the temporal coherence of the main pulse and generates a noisy structure around the main pulse. A degradation of a few orders of magnitude is estimated with the random spectral phase noise.
\end{abstract}

Key Words: Ultra-high intensity lasers, Chirped-pulse amplification (CPA), temporal contrast, Ti:sapphire lasers, Diffraction and gratings

\section{Introduction}

With the remarkable progress of the chirped-pulse amplification (CPA) technique, ${ }^{1)}$ petawatt (PW)-class Ti:sapphire laser systems have been constructed worldwide ${ }^{2-4)}$ and the focused intensity has reached $10^{20}-10^{22} \mathrm{~W} / \mathrm{cm}^{2}$ in small scale laboratories. ${ }^{5,6}$ Recently, based on the demonstration of PW laser systems, some $10 \mathrm{PW}$ Ti:sapphire laser systems are being constructed in France, ${ }^{7)}$ China, ${ }^{8)}$ and the European Extreme Light Infrastructure.9) In parallel, the optical parametric chirped-pulse amplification (OPCPA) technique ${ }^{10,11)}$ is also being developed as an alternative way to potentially generate peak power in the PW regime ${ }^{12)}$ and beyond. ${ }^{13,14)}$ Based on ultra-intense and ultra-fast lasers, many exciting opportunities for studying laser-matter interactions in the relativistic dominated regime have become possible. ${ }^{6,15-19)}$

For relativistic laser-matter interactions, increased peak intensities require a commensurate improvement in temporal contrast. In particular, the contrast is an important factor in the application of an ultra-high intensity laser to solid-target experiments because the pre-pulse can generate unwanted plasma before the main pulse arrives at the target. Usually, there exist three kinds of pre-pulses in ultra-high intensity lasers: femtosecond pre-pulse, amplified spontaneous emission (ASE), and pedestal. The contrast of the femtosecond prepulse and ASE are improved by several techniques such as the usage of Pockels cells, ${ }^{20)}$ double CPA architecture ${ }^{4)}$ and so on. ${ }^{21-23)}$ With the significant enhancement of the contrast in ASE, the pedestal around the main pulse, which has been buried by ASE and has been invisible so far in the low ASE contrast case, was found and is clearly independent ASE. The pedestal is a fundamental limiting factor in relativistic experiments and it should be investigated.

Here, we discuss quantitatively the random spectral phase noise (RSPN) effect on the pedestal around the main pulse in the temporal contrast. The surface roughness of tens of nano- meters on the stretcher and compressor optics causes RSPN, which reduces the temporal coherence of the main pulse and generates a noisy structure around the main pulse. Based on the detailed analysis, it was found that RSPN makes it possible to produce a pedestal structure around the main pulse and causes a degradation of a few orders of magnitude in the contrast.

\section{Random spectral phase noise}

In CPA lasers, a laser beam is spectrally resolved on optics in a stretcher and compressor, shown in Fig. 1. Thus, the surface roughness causes the relative phase shift at each wavelength and is directly converted to RSPN. This means that the surface quality of the stretcher and compressor optics such as mirror and gratings is important for spectral phase noise, which reduces the temporal contrast of the main pulse and generates a noisy structure on the sides of the main pulse.

\section{Results and discussion}

In order to understand the effect of RSPN on the temporal contrast induced by the optics surface roughness in the stretcher and compressor, we have measured the surface quality of all of the gratings and the roof mirrors which were used in the previous J-KAREN laser system. ${ }^{4}$ However, the measurement of the convex and concave mirrors is technically difficult. Therefore, we have not considered the effect of RSPN with these mirrors. The optics qualities have been measured by using a commercial interferometer (ZYGO, GPI XP HR, Canon Inc.). Figure 2 shows the representative measured surface roughness of the roof mirror in the stretcher and the grating in the compressor. The surface roughness is $23 \mathrm{~nm}$ in PV (peak to valley) for the roof mirror and $41 \mathrm{~nm}$ PV for the grating in the compressor even though the noise structure is different. We have measured the surface roughness of the 
a

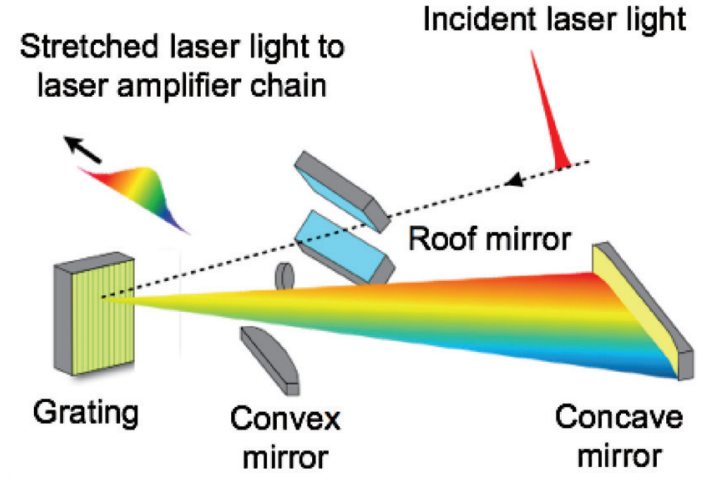

b

\section{Stretched laser light from Output laser light laser amplifier chain}
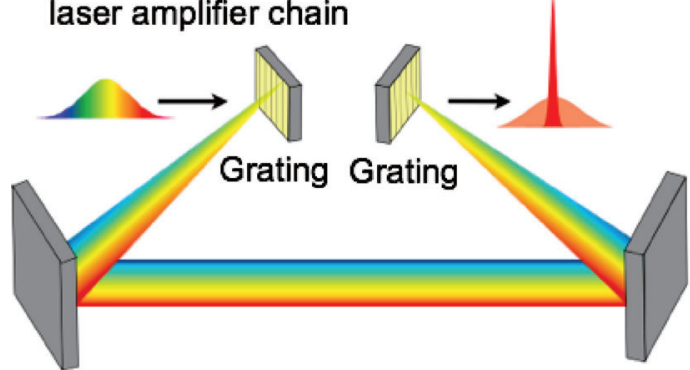

Grating

Grating

Fig. 1 Stretcher (a) and compressor (b) in chirped-pulse amplification (CPA) laser system. A laser beam is spectrally resolved on optics.
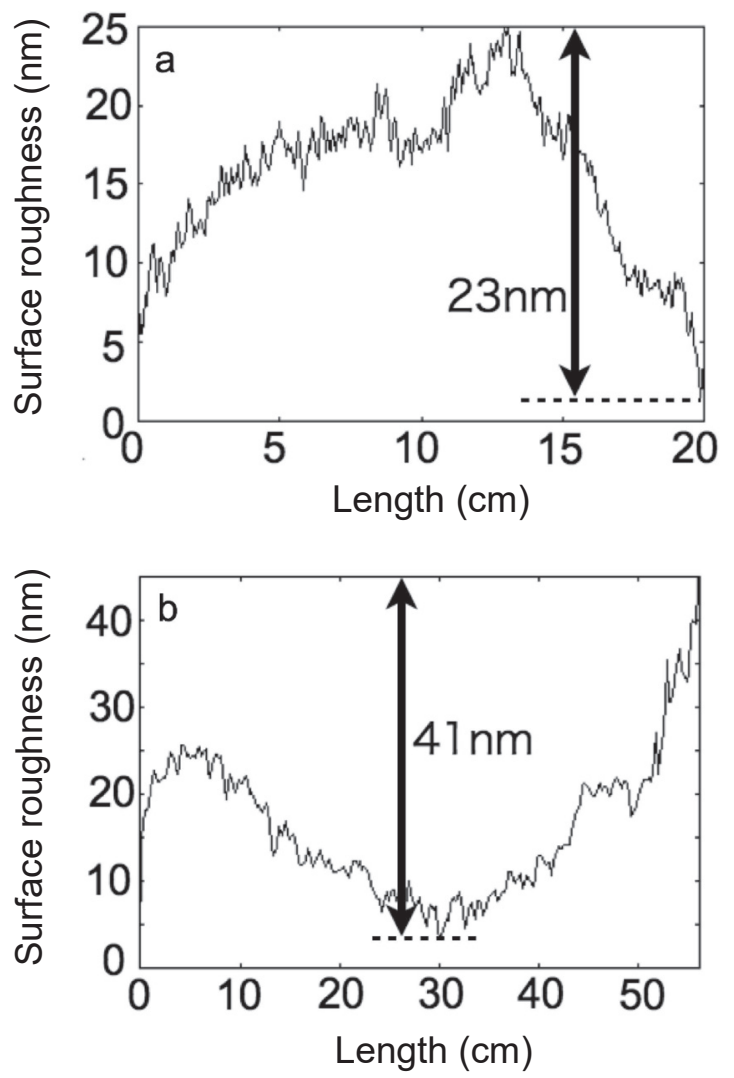

Fig. 2 Measurement of representative surface roughness of roof mirror (a) and grating in compressor (b). optics, considering the portion in which the laser is reflected. As can be seen clearly, the optics has high-frequency surface modulations that can cause RSPN in a pulse stretcher and compressor.

Having obtained the surface roughness, the temporal contrast $C(t)$ can be expressed using the Fourier-inverse-transformation as follows:

$$
C(t)=\left(\int{\sqrt{I(\lambda) e^{i}}}^{i\left(\frac{2 \pi c}{\lambda} t+\delta(\lambda)\right)} d \lambda\right)^{2}
$$

$\operatorname{RSPN} \delta(\lambda)$ can be expressed as follows (as seen in Fig. 3):

$$
\delta(\lambda)=\Delta D \times \frac{2 \pi}{\lambda}
$$

where $I(\lambda)$ is spectral intensity, and $c$ is the speed of light. Based on eq. (1), we are able to quantitatively analyze the impact of the optics surface roughness in the stretcher and the compressor on the temporal contrast pedestal. We have evaluated the contrast pedestal two dimensionally, taking the finite beam size in the stretcher and the compressor into account. We have assumed a typical Fourier-transform-limited 35 fs pulse with a central wavelength of $810 \mathrm{~nm}$.

Figure 4 shows the calculated RSPN caused by the stretcher and the compressor. The RSPN is $1.7 \mathrm{rad}$ in PV. Using this RSPN, we have calculated the temporal contrast. Figure 5 shows the temporal contrast with and without RSPN. As can be seen, the calculation predicts that the RSPN can degrade the contrast pedestal greater than two orders of magnitude by the optics in the stretcher and the compressor. This has demonstrated that the surface roughness of the optics is the main source of the contrast pedestal and also underpinned the principle behind the contrast pedestal of ultra-high

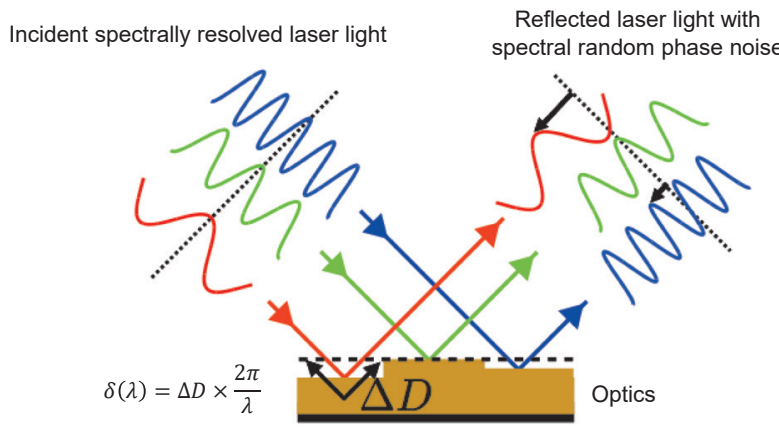

Fig. 3 Origin of spectral random phase noise by surface roughness of the stretcher and compressor.

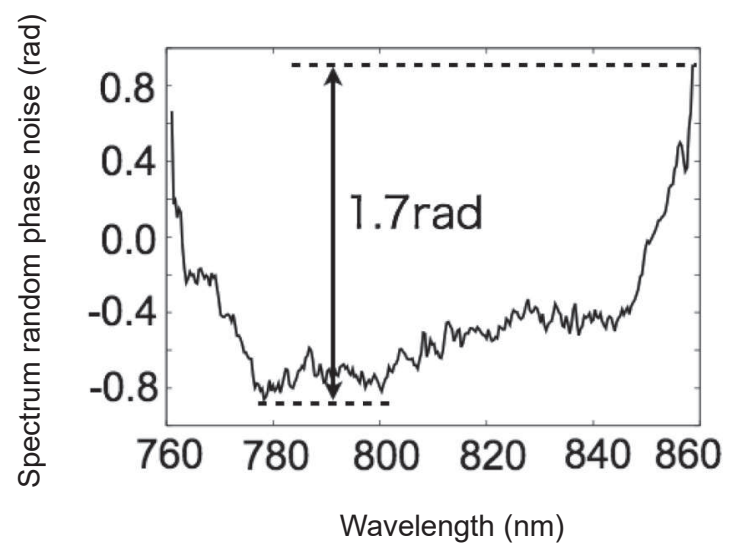

Fig. 4 Calculated random spectral phase noise of the stretcher and compressor. 


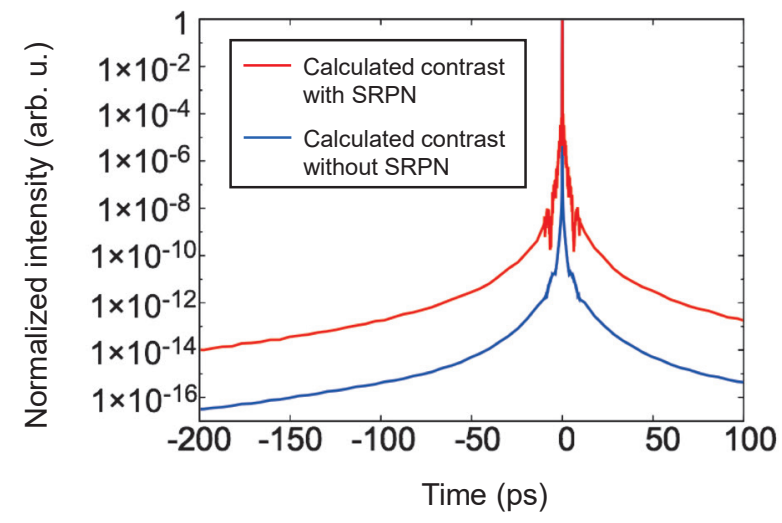

Fig. 5 Calculated temporal contrast with and without random spectral phase noise.

intensity laser pulses. In principle, based on the optical geometry of the stretcher and compressor, and gratings and mirrors to be used, this technique could be employed to predict the contrast pedestal of ultra-high intensity CPA laser pulses by measuring the optics surface roughness precisely, even before the actual system is constructed.

\section{Conclusion}

RSPN can be a good explanation for the contrast pedestal. Therefore, we concluded that the RSPN generated in the optics in the stretcher and the compressor is the most probable factor causing the pedestal. As the ASE contrast gets even higher, for example, more than $10^{12}$ for the experiment with the focused intensity of over $10^{22} \mathrm{~W} / \mathrm{cm}^{2}$, the RSPN becomes more important because the pedestal could exist for more than tens of ps around the main pulse.

\section{Acknowledgement}

This work was partially funded by JSPS KAKENHI Grant Number JP16H03911. The authors acknowledge contributions by the Kansai Photon Science Institute staff at National Institute for Quantum and Radiological Science and Technology for their support of this work. One of the authors, H. Kiriyama especially thanks J. Koga for stimulating discussion and encouragement.

\section{References}

1) D. Strickland and G. Mourou: Opt. Commun. 56 (1985) 219.

2) T. Jun Yu, S. K. Lee, J. H. Sung, J. W. Yoon, T. M. Jeong, and J. Lee: Opt. Exp. 40 (2012) 10807.

3) S. Laux, F. Lureau, C. Radier, O. Chalus, F. Caradec, O.
Casagrande, E. Pourtal, C. Simon-Boisson, F. Soyer, and P. Lebarny: Opt. Lett. 37 (2012) 1913.

4) H. Kiriyama, M. Mori, A. S. Pirozhkov, K. Ogura, A. Sagisaka, A Kon, T. Zh. Esirkepov, Y. Hayashi, H. Kotaki, M. Kanasaki, et al.: IEEE Sel. Topics J. Quantum Electron. (Invited Paper) 21 (2015) 1601118.

5) S.-W. Bahk, P. Rousseau, T. A. Planchon, V. Chvykov, G. Kalintchenko, A. Maksimchuk, G. A. Mourou, and V. Yanovsky: Opt. Lett. 29 (2004) 2837.

6) K. Ogura, M. Nishiuchi, A. S. Pirozhkov, T. Tanimoto, A. Sagisaka, T. Zh. Esirkepov, M. Kando, T. Shizuma, T. Hayakawa, H. Kiriyama, et al.: Opt. Lett. 37 (2012) 2868.

7) C. Le Blanc, D. N. Papadopoulos, G. Ch'eriaux, P. Georges, J.-P. Zou, G. Mennerat, F. Druon, A. Pellegrina, P. Ramirez, F. Giambruno, et al.: Rev. Laser Eng. 42 (2014) 127.

8) Y. Chu, Z. Gan, X. Liang, L. Yu, X. Lu, C. Wang, X. Wang, L. Xu, H. Lu, D. Yin, et al.: Opt. Lett. 40 (2015) 5011.

9) S. Weber, S. Bechet, S. Borneis, L. Brabec, M. Bucka, E. Chacon-Golcher, M. Ciappina, M. DeMarco, A. Fajstavr, K. Falk, et al.: Matter and Radiation at Extremes 2 (2017) 149.

10) A. Dubietis, G. Jonuauskas, and A. Piskarskas: Opt. Commun. 88 (1992) 437.

11) I. N. Ross, P. Matousek, M. Towrie, A. J. Langley, and J. L. Collier: Opt. Commun. 144 (1997) 125

12) L. Yu, X. Liang, L. Xu, W. Li, C. Peng, Z. Hu, C. Wang, X. Lu, Y. Chu, Z. Gan, et al.: Opt. Lett. 40 (2015) 3412.

13) V. V. Lozhkarev, G. I. Freidman, V. N. Ginzburg, E. V. Katin, E. A. Khazanov, A. V. Kirsanov, G. A. Luchinin, A. N. Mal'shakov, M. A. Martyanov, O. V. Palashov, et al.: Laser Phys. Lett. 4 (2007) 1.

14) M. Galimberti, I. O. Musgrave, A. Boyle, C. Hernandez-Gomez, T. B. Winstone, W. Shaikh, A. Wyatt, D. Pepler, I. N. Ross, A. Lyachev, et al.: Rev. Laser Eng. 42 (2014) 137.

15) Y. Fukuda, H. Sakaki, M. Kanasaki, A. Yogo, S. Jinno, M. Tampo, A. Y. Faenov, T. A. Pikuz, Y. Hayashi, M. Kando, et al.: Radiat. Meas. 50 (2013) 92.

16) Y. Hayashi, A. S. Pirozhkov, M. Kando, Y. Fukuda, A. Faenov, K. Kawase, T. Pikuz, T. Nakamura, H. Kiriyama, H. Okada, et al.: Opt. Lett. 36 (2011) 1614.

17) A. S. Pirozhkov, M. Kando, T. Zh. Esirkepov, P. Gallegos, H. Ahmed, E. N. Ragozin, A. Y. Faenov, T. A. Pikuz, T. Kawachi, A. Sagisaka, et al.: Phys. Rev. Lett. 108 (2012) 135004.

18) Z. Zhang, H. Nishimura, T. Namimoto, S. Fujioka, Y. Arikawa, M. Nishikino, T. Kawachi, A. Sagisaka, H. Hosoda, S. Orimo, et al.: Rev. Sci. Instrum. 83 (2012) 053502.

19) Ya. Faenov, I. Yu. Skobelev, T. A. Pikuz, S. A. Pikuz, V. E. Fortov, Y. Fukuda, Y. Hayashi, A. Pirozhkov, H. Kotaki, T. Shimomura, et al.: Laser Particle Beams 30 (2012) 481.

20) M. Nantel, J. Itatani, A.-C. Tien, J. Faure, D. Kaplan, M. Bouvier, T. Buma, P. V. Rompay, J. Nees, P. P. Pronko, et al.: IEEE Sel. Topics J. Quantum Electron. 4 (1998) 449.

21) I. J. Kim, I.W. Choi, S.K. Lee, K. A. Janulewicz, J. H. Sung, T. J.Yu, H. T. Kim, H. Yun, T. M. Jeong, and J. Lee: Appl. Phys. B, Lasers Opt. 104 (2011) 81.

22) R. Toth, S. Fourmaux, T. Ozaki, M. Servol, J. C. Kieffer, R. E. Kincaid Jr., and A. Krol: Phys. Plasmas 14 (2007) 053506.

23) J. Liu, K. Okamura, Y. Kida, and T. Kobayashi: Opt. Exp. 18 (2010) 22245. 